\title{
Ultrasound guided injections of botulinum toxin type $A$ into stellate ganglion to treat insomnia
}

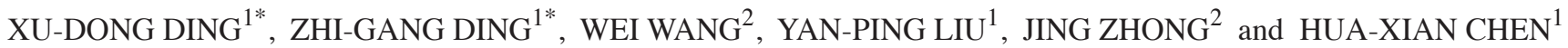 \\ Departments of ${ }^{1}$ Rehabilitation Medicine and ${ }^{2}$ Ultrasound Imaging, Xiangyang No. 1 People's Hospital, \\ Hubei University of Medicine, Xiangyang, Hubei 441000, P.R. China
}

Received July 20, 2016; Accepted June 2, 2017

DOI: $10.3892 /$ etm.2017.4612

\begin{abstract}
The aim of the study was to investigate the curative effect of botulinum toxin type A (BTX-A) injection into stellate ganglion under ultrasound guidance in patients suffering from insomnia. From October 2015 to April 2016, 48 patients suffering from insomnia were enrolled in this study. Patients were divided into 2 groups using a random digital grouping method: i) Control group (24 cases), and ii) treatment group (24 cases). Patients in the control group received $1 \mathrm{mg}$ oral estazolam 30 min before sleep every night, while patients in the treatment group received BTX-A injection in bilateral stellate ganglions under ultrasound guidance. Curative effect evaluation was carried out after treatment. The international Pittsburgh Sleep Quality Index (PSQI) and polysomnogram (PSG) were evaluated in the two groups before and after treatment. The total effective rate was obviously higher in the treatment group. The PSQI score and the results of the PSG indicated that the insomnia situation improved in both groups. However, compared with the control group, the treatment group had a more significant improvement. In conclusion, BTX-A injection in stellate ganglion was a relatively easy and effective way to treat insomnia without any notable adverse reactions.
\end{abstract}

\section{Introduction}

Insomnia is a common form of sleep disorder characterized by difficulty in falling and staying asleep. In individuals suffering from this condition, sleep quality or quantity cannot reach normal requirement (1). Insomnia can be caused by psychiatric and medical conditions, and unhealthy sleep habits. Chronic insomnia may seriously affect patients' physical

Correspondence to: Dr Hua-Xian Chen, Department of Rehabilitation Medicine, Xiangyang No. 1 People's Hospital, Hubei University of Medicine, 15 Jiefang Road, Xiangyang, Hubei 441000, P.R. China

E-mail: chen_huaxian1@163.com

"Contributed equally

Key words: insomnia, botulinum toxin type A, ultrasound guidance, stellate ganglion as well as psychological health and can lead to decrease of attention, judgment impairment, loss of memory, anxiety and depression (1). Unhealthy lifestyles and sleep habits can create insomnia or exacerbate the insomnia problem worse.

In the treatment of insomnia, non-benzodiazepine hypnotic drugs are the first line of management followed by benzodiazepines, amitryptiline and antihistaminics. However, these medications can create short- and long-term adverse effects, including drug dependence, addiction and withdrawal reaction (2). In the present study, we investigated the curative effect of botulinum toxin type A (BTX-A) on patients suffering from insomnia, by injecting BTX-A into stellate ganglion under ultrasound guidance, and obtained promising results.

\section{Materials and methods}

General data. From October 2015 to April 2016, 48 cases of insomnia were enrolled in the present study. There were 20 males and 28 females (Table I). All the patients suffered from different levels of insomnia symptoms, including difficulty to fall asleep, drowsiness, early awakening, anxiety and physical problems. Inclusion criteria for the study were: i) Patients who complied with the insomnia diagnostic criteria provided by the sleep disorder group of Neurology Branch of Chinese Medical Association, and were given confirmed diagnosis by more than two specialists; ii) patients with Pittsburgh Sleep Quality Index (PSQI) score $\geq 14$; iii) patients not taking hypnotic drugs within 4 weeks prior to their enrolment; and iv) patients with a maximum of 9 years of education.

Exclusion criteria for the study were: i) Patients with transitory insomnia or insomnia caused by environmental changes; ii) known alcoholics and drug addicts; iii) pregnant and lactating women, and those suffering from serious physical illnesses and neuropsychic diseases; and iv) patients who did not complete the follow-ups.

All the patients signed informed consent forms. The study was approved by the Ethics Committee of Xiangyang No. 1 People's Hospital, Hubei University of Medicine (Hubei, China).

Treatment methods. Patients were divided into 2 groups using a random digital grouping method: i) Control group (24 cases) and ii) treatment group (24 cases). Patients in the control group received $1 \mathrm{mg}$ oral estazolam $30 \mathrm{~min}$ before sleep every night, while patients in the treatment group received BTX-A injection 
Table I. Comparison of general data in patients.

\begin{tabular}{|c|c|c|c|c|}
\hline Items & Control group & Treatment group & Test value & P-value \\
\hline Case & 22 & 23 & $\left(\mathrm{t} / \chi^{2}\right)$ & \\
\hline Age (years) & $55.62 \pm 8.35$ & $56.54 \pm 9.23$ & -0.350 & 0.728 \\
\hline Sex (male) & $9(67.3 \%)$ & $10(67.5 \%)$ & 0.030 & 0.862 \\
\hline Course of disease (years) & $4.24 \pm 1.37$ & $4.10 \pm 1.18$ & -1.000 & 0.715 \\
\hline Diabetes & $5(30.8 \%)$ & $4(27.5 \%)$ & 0.200 & 0.655 \\
\hline Hypertension & $8(23.1 \%)$ & $9(20.0 \%)$ & 0.037 & 0.848 \\
\hline Heart disease & $3(48.1 \%)$ & $2(50.0 \%)$ & 0.278 & 0.598 \\
\hline Education level (years) & $12.62 \pm 2.38$ & $12.21 \pm 2.87$ & -1.000 & 0.606 \\
\hline PSQI total points & $17.43 \pm 2.42$ & $16.38 \pm 2.32$ & -1.000 & 0.145 \\
\hline
\end{tabular}

PSQI, Pittsburgh Sleep Quality Index.

in bilateral stellate ganglions under ultrasound guidance. For the injection, patients were required to be in a supine position with contralateral head turning. After routine disinfection of skin, puncture needle no. 7 was inserted perpendicularly through the sixth vertebral transverse. The needle was then withdrawn a little, needle tail inclined to the head end at $45^{\circ}$, and the needle head was passed through the front of the sixth vertebral transverse and advanced $\sim 1 \mathrm{~cm}$ towards the direction of the seventh vertebral transverse. In case of resorption without blood or cerebrospinal fluid, local anesthetics were administered.

\section{Observation methods}

PSQI. PSQI scale included 7 indexes: i) Sleep quality; ii) time for falling asleep; iii) sleep time; iv) sleep efficiency; v) sleep disorder; vi) hypnotic drugs; and vii) daytime functions. Each index was scored as 0-3 scores, with 0 for no difficulty, 1 for mild difficulty, 2 for moderate difficulty and 3 for severe difficulty. Total PSQI scores were the sum of all the scores, with the total score ranging from 0 to 21 scores. A total score of $\geq 7$ represented the existence of sleep disorder and higher PSQI scores were associated with lower sleep quality (3).

Polysomnogram. The Alice 4 polysomnography system (Respironics Inc., Murrysville, PA, USA) was used to conduct polysomnogram (PSG) detection. EEG lead used C4/A1 and C5/A2, EMG electrodes were placed at chin, and EOG electrodes were placed at $1 \mathrm{~cm}$ from the outer canthus of both eyes. Patients were tested separately in a quiet, comfortable and lightproof sleep lab. PSGs were recorded starting from the second night. When the sleep record did not reflect the situations of usual sleep, data were discarded (4). Sleep staging criteria referred to the International Standards for Polysomnographic Recording and Staging formulated by American Rechtschaffen and Kales (5). The observation indexes included: i) Sleep process; sleep latency (SL), arousal time (AT), arousal wake up of time (AWT), rapid eye movement (REM) latency (RL), sleep efficiency (SE) and total sleep time (TST); and ii) sleep structure included no REM (NREM) sleep and REM sleep. NREM included four stages, namely S1, S2, S3 and S4. The percentage of each stage from the total sleep time was calculated. Both S3 and S4 belonged to deep sleep and were combined into one.
Evaluation of therapeutic effects. According to the subjective feelings of patients, the therapeutic effects were judged in accordance with the evaluation standards for therapeutic effects on insomnia specified in the Amended Draft of Standards for Therapeutic Effects of Mental Diseases (6). Healing was achieved when the sleep condition was significantly improved, when the patient slept 6-8 h sleep/night and enjoyed deep sleep and was full of energy during the next day. We considered that the treatment had a significant effect when the patient slept 3-5 h sleep/night and associated symptoms were disappeared or obviously reduced. Treatment was considered effective when sleep condition was improved and the sleep time was prolonged up to $2 \mathrm{~h}$. When sleep condition and associated symptoms were not improved at all or the improvement failed to reach the standards to be considered effective, treatment was considered to be non-effective.

Observation results. Patients in both groups were subjected to PSQI assessment and PSG test before and after treatment. An assessment of curative effect of treatment was carried out after treatment.

Statistical analysis. We used SPSS 17.0 software (Chicago, IL, USA) for our statistical analysis. Measurement data were expressed as mean \pm SD and comparison between groups was completed with the t-test. $\mathrm{P}<0.05$ was considered to indicate a statistically significant difference.

\section{Results}

Comparison of general materials. From 48 patients who were enrolled in this study, three cases were excluded after the beginning of the experiments due to various reasons. Consequently, the study 22 cases in the control group and 23 cases in the treatment group were continued. Differences in patients' general characteristics in the two groups had no statistical significance and were comparable (Table II).

Curative effect evaluation. After being treated for 4 weeks, the total effective rate in the treatment group was obviously higher than that of the control group and the difference was statistically significant $(\mathrm{P}<0.05)$. The total effective rates 
Table II. Comparison of postoperative curative effect in the two groups.

\begin{tabular}{lccccc}
\hline Groups & Cured & Markedly effective & Effective & Ineffective & Effective rate (\%) \\
\hline Control (22 cases) & 2 & 5 & 8 & 7 & 68.18 \\
Treatment (23 cases) & 5 & 9 & 6 & 3 & 86.95 \\
\hline
\end{tabular}

Table III. Comparison of PSQI score in the two groups before and after treatment (mean \pm SD).

\begin{tabular}{|c|c|c|c|c|c|}
\hline Groups & Case & PSQI total points & Sleep quality & Time for falling asleep & Sleep time \\
\hline \multicolumn{6}{|l|}{ Control } \\
\hline Before treatment & 22 & $17.43 \pm 2.42$ & $2.61 \pm 0.46$ & $2.65 \pm 0.59$ & $2.56 \pm 0.55$ \\
\hline After treatment & 22 & $13.06 \pm 1.82^{\mathrm{a}}$ & $1.82 \pm 0.38^{\mathrm{a}}$ & $1.93 \pm 0.48^{\mathrm{a}}$ & $2.18 \pm 0.38^{\mathrm{a}}$ \\
\hline \multicolumn{6}{|l|}{ Treatment } \\
\hline Before treatment & 23 & $16.38 \pm 2.32$ & $2.58 \pm 0.37$ & $2.59 \pm 0.45$ & $2.62 \pm 0.52$ \\
\hline After treatment & 23 & $10.15 \pm 1.90^{\mathrm{a}, \mathrm{b}}$ & $1.33 \pm 0.35^{\mathrm{a}, \mathrm{b}}$ & $1.63 \pm 0.32^{\mathrm{a}, \mathrm{b}}$ & $1.74 \pm 0.48^{\mathrm{a}, \mathrm{b}}$ \\
\hline Groups & Case & Sleep efficiency & Sleep disorder & Hypnotic drugs & Daytime function \\
\hline \multicolumn{6}{|l|}{ Control } \\
\hline Before treatment & 22 & $2.52 \pm 0.48$ & $2.51 \pm 0.66$ & $2.65 \pm 0.37$ & $2.66 \pm 0.35$ \\
\hline After treatment & 22 & $2.02 \pm 0.39^{\mathrm{a}}$ & $2.02 \pm 0.38^{\mathrm{a}}$ & $2.33 \pm 0.28^{\mathrm{a}}$ & $1.98 \pm 0.48^{\mathrm{a}}$ \\
\hline \multicolumn{6}{|l|}{ Treatment } \\
\hline Before treatment & 23 & $2.62 \pm 0.37$ & $2.64 \pm 0.47$ & $2.59 \pm 0.35$ & $2.60 \pm 0.47$ \\
\hline After treatment & 23 & $1.60 \pm 0.41^{\mathrm{a}, \mathrm{b}}$ & $1.33 \pm 0.35^{\mathrm{a}, \mathrm{b}}$ & $1.83 \pm 0.42^{\mathrm{a}, \mathrm{b}}$ & $1.59 \pm 0.38^{\mathrm{a}, \mathrm{b}}$ \\
\hline
\end{tabular}

Compared within group before and after treatment, ${ }^{\mathrm{a}} \mathrm{P}<0.05$; comparison of the treatment group with the control group, ${ }^{\text {b }}<0.05$. PSQI, Pittsburgh Sleep Quality Index.

Table IV. Comparison of sleep process indicators in the two groups before and after treatment (mean \pm SD).

\begin{tabular}{|c|c|c|c|c|c|c|}
\hline Groups & $\mathrm{SL}(\min )$ & AWT (min) & AT (time) & $\mathrm{RL}$ (min) & SE $(\%)$ & TST (min) \\
\hline \multicolumn{7}{|l|}{ Control (n=22) } \\
\hline Before treatment & $60.92 \pm 22.18$ & $120.73 \pm 70.50$ & $7.16 \pm 2.41$ & $112.83 \pm 32.39$ & $61.43 \pm 11.43$ & $234.28 \pm 62.24$ \\
\hline After treatment & $42.39 \pm 21.35^{\mathrm{a}}$ & $102.25 \pm 60.48$ & $5.24 \pm 2.18^{\mathrm{a}}$ & $81.08 \pm 37.48^{\mathrm{a}}$ & $78.45 \pm 7.08^{\mathrm{a}}$ & $302.59 \pm 58.93^{\mathrm{a}}$ \\
\hline \multicolumn{7}{|l|}{ Treatment $(n=23)$} \\
\hline Before treatment & $59.82 \pm 23.16$ & $121.78 \pm 72.57$ & $7.47 \pm 2.26$ & $110.80 \pm 32.43$ & $60.42 \pm 10.12$ & $232.42 \pm 66.73$ \\
\hline After treatment & $25.78 \pm 10.08^{\mathrm{a}, \mathrm{b}}$ & $48.5 \pm 19.40^{\mathrm{a}, \mathrm{b}}$ & $3.55 \pm 2.42^{\mathrm{a}, \mathrm{b}}$ & $65.62 \pm 23.68^{\mathrm{a}, \mathrm{b}}$ & $89.53 \pm 6.85^{\mathrm{a}, \mathrm{b}}$ & $385.16 \pm 52.27^{\mathrm{a}, \mathrm{b}}$ \\
\hline
\end{tabular}

Compared within group before and after treatment, ${ }^{\mathrm{a}} \mathrm{P}<0.05$; compared with the treatment and control groups, ${ }^{\text {b }}<0.05$. $\mathrm{SL}$, sleep latency; AWT, arousal wake up of time; AT, arousal time; RL, rapid eye movement latency; SE, sleep efficiency; TST, total sleep time.

in the treatment and control groups were 86.95 and $68.18 \%$, respectively (Table II).

Comparison of PSQI score of patients in the two groups before and after treatment. After 4 weeks of treatment, the PSQI score indicated that the insomnia situation was improved in both groups. However, compared with the control group, the treatment group had a more significant improvement. The difference was statistically significant $(\mathrm{P}<0.05)$ (Table III).
PSG results. After 4 weeks of treatment, PSG monitoring results showed that the insomnia condition improved in both groups, but the improvement in the treatment group was more obvious. The difference was statistically significant $(\mathrm{P}<0.05)$ (Tables IV and V).

\section{Discussion}

Sleep disorder refers to a reduction of sleep time or sleep quality caused by difficulties associated with falling asleep, 
Table V. Comparison of sleep structure index in the two groups before and after treatment (\%, mean \pm SD).

\begin{tabular}{lcccc}
\hline & \multicolumn{3}{c}{ NREM } & REM \\
\cline { 2 - 3 } Groups & $\mathrm{S} 1$ & $\mathrm{~S} 2$ & $\mathrm{~S} 3+\mathrm{S} 4$ & \\
\hline Control (n=22) & & & \\
Before treatment & $23.81 \pm 7.18$ & $55.73 \pm 10.50$ & $9.16 \pm 5.01$ & $11.83 \pm 2.39$ \\
After treatment & $17.39 \pm 6.05^{\mathrm{a}}$ & $20.12 \pm 11.48^{\mathrm{a}}$ & $13.24 \pm 4.11^{\mathrm{a}}$ & $15.08 \pm 3.48^{\mathrm{a}}$ \\
Treatment (n=23) & & & & \\
Before treatment & $27.82 \pm 7.16$ & $56.48 \pm 11.37$ & $9.17 \pm 4.06$ & $13.80 \pm 7.43$ \\
After treatment & $8.78 \pm 3.08^{\mathrm{a}, \mathrm{b}}$ & $46.22 \pm 8.42^{\mathrm{a}, \mathrm{b}}$ & $19.35 \pm 6.02^{\mathrm{a}, \mathrm{b}}$ & $21.62 \pm 3.68^{\mathrm{a}, \mathrm{b}}$ \\
\hline
\end{tabular}

Compared within group before and after treatment, ${ }^{a} \mathrm{P}<0.05$; compared with the treatment and control groups, ${ }^{\mathrm{b}} \mathrm{P}<0.05$. NREM, no rapid eye movement; REM, rapid eye movement.

easy awakening, early awakening and difficulty of getting back to sleep. Numerous factors may lead to sleep disorder, but the most important factors are somatic factors, drug factors, environmental factors, mental factors and human biological clock factors (7). The direct cause of sleep disorder is the abnormities of neurotransmitter, endocrine and other systems caused by cerebral cortex and vegetative nerve dysfunctions. Sleep disorder treatment is usually focused on recovering and coordinating the function of cerebral cortex as well as inhibiting the excessive hyperexcitability of vegetative nerves. Currently, sleep disorder treatment involves drug therapy, physical therapy, traditional Chinese medicine acupuncture therapy, stellate ganglion block and psychological therapy. There are several problems associated with long-term administration of sedative-hypnotic drugs, including dizziness, loss of strength, drug dependence, addiction and withdrawal reaction. Although single stellate ganglion block showed to be a good treatment for insomnia, its long course of treatment and the high risk of recurrence have caused some concerns. The therapeutic effects of physical, acupuncture and psychological therapies on improving insomnia are uncertain and the risk of recurrence exists.

Stellate ganglion, also known as cervical and thoracic sympathetic ganglion, is mainly fused by cervical sympathetic ganglion 7 and 8, and thoracic sympathetic ganglion 1. A number of studies proved that stellate ganglion is involved in adjusting vegetative nerves, inhibiting nervous excitation, relax mental stress, adjusting the relaxation balance of automatic nervous system, increasing cerebral blood flow and improving sleep $(8,9)$.

In clinic, BTX-A is used for the treatment of strabismus, eye muscle spasm, facial tics, muscle spasm and has obtained good effects. It has been shown that BTX-A can inhibit calcium-dependent $\mathrm{ACh}$ transportation and lower the mobility of lipid membrane. Thus, BTX-A can disturb nerve conduction by inhibiting the translocation of synaptic vesicles, the fusion with presynaptic membrane and the release of ACh (10).

In this study, we observed the effects of BTX-A on insomnia by injecting BTX-A in bilateral stellate ganglions. Both subjective evaluation (PSQI and evaluation of therapeutic effects) and objective detection indexes (PSG) results in our treatment group were significantly superior to those of the control group.
These results suggested that the injection of BTX-A in stellate ganglion improved the sleep situations of patients and had definite curative effect on insomnia. The exact mechanism of this curative effect remains unclear, but some suggestions have been made: i) BTX-A may improve the situation by relieving cerebral angiospasm and adjust the balance of vasomotor function. It increases the energy reserves in cerebral cortex nerve cells by boosting aerobic metabolism of brain (11). ii) BTX-A may adjust vegetative nerve functional disturbance caused by the increase of sympathetic nerve activity, improve sympathetic-vagus unbalance and lower the level of plasma norepinephrine caused by it. Thus BTX-A can improve the function of vegetative nerve (12). iii) It has an active two-way regulation function in hypothalamus and generates significant effects on the secretion of hypophyseal hormones. It can regulate the function of automatic nervous, endocrine and immune systems. By regulating the hypothalamic function, BTX-A can maintain the stability of the internal environment and ameliorate the function of the endocrine, immune and vegetative nerve systems. By maintaining the stability of these systems, BTX-A can help patients with insomnia to return to their normal biological clock set up (13). iv) Pineal gland, under the control of sympathetic nerve, periodically secretes melatonin during day and night, which affects sleep and wakefulness. The increased tonicity of sympathetic nerve causes the dysrhythmia of melatonin secretion. The secretion of melatonin is adjusted to correct the disorders of circadian rhythm. Uchida et al reported that an increase in the tonicity of sympathetic nerve caused the dysrhythmia of melatonin secretion and stellate ganglion block (5). This situation led to a reduction in sympathetic nerve tonicity and adjusted sleep condition. v) BTX-A inhibits sympathicoadrenal system and lowers the level of epinephrine, cortisol as well as angiotensin II (14) and also adjusts autonomic nerve function.

The surrounding anatomy of the stellate ganglion is very complex. Several key structures such as apex pulmonis, scalene muscle, arteria subclavia, common carotid artery, vertebral artery, internal thoracic artery, inferior thyroid artery, internal jugular vein, brachiocephalic vein, vagus nerve and phrenic nerve can be found around this ganglion. Utmost care must be taken to prevent damage during the injection. In case of carelessness, complications such as local hematoma, pneumothorax 
or hemopneumothorax, recurrent laryngeal nerve block, upper extremity palsy and stellate ganglion injuries may occur (15). In this study, all the injections were completed under ultrasound guidance without any adverse reaction.

In summary, results obtained from our study demonstrated that BTX-A injection in stellate ganglion under ultrasound guidance was a relatively easy and effective way to treat insomnia without any notable adverse reaction.

\section{References}

1. Gillin JC, Spinweber CL and Johnson LC: Rebound insomnia: a critical review. J Clin Psychopharmacol 9: 161-172, 1989.

2. Huedo-Medina TB, Kirsch I, Middlemass J, Klonizakis M and Siriwardena AN: Effectiveness of non-benzodiazepine hypnotics in treatment of adult insomnia: meta-analysis of data submitted to the Food and Drug Administration. BMJ 345 e8343, 2012.

3. Tsai PS1, Wang SY, Wang MY, Su CT, Yang TT, Huang CJ and Fang SC: Psychometric evaluation of the Chinese version of the Pittsburgh Sleep Quality Index (CPSQI) in primary insomnia and control subjects. Qual Life Res 14: 1943-1952, 2005.

4. Roehrs TA, Randall S, Harris E, Maan R and Roth T: MSLT in primary insomnia: stability and relation to nocturnal sleep. Sleep 34: 1647-1652, 2011.

5. Uchida K, Tateda T and Hino H: Novel mechanism of action hypothesized for stellate ganglion block related to melatonin. Med Hypotheses 59: 446-449, 2002.

6. Burbach JP, Hellemons AJ, Hoekman M, Grant P and Pant HC: The stellate ganglion of the squid Loligo pealeii as a model for neuronal development: expression of a POU Class VI homeodomain gene, Rpf-1. Biol Bull 201: 252-254, 2001.
7. Taylor DJ, Lichstein KL and Durrence HH: Insomnia as a health risk factor. Behav Sleep Med 1: 227-247, 2003.

8. Chen YQ, Jin XJ, Liu ZF and Zhu MF: Effects of stellate ganglion block on cardiovascular reaction and heart rate variability in elderly patients during anesthesia induction and endotracheal intubation. J Clin Anesth 27: 140-145, 2015.

9. Hicky A, Hanling S, Pevney E, Allen R and McLay RN: Stellate ganglion block for PTSD. Am J Psychiatry 169: 760, 2012.

10. Welch MJ, Purkiss JR and Foster KA: Sensitivity of embryonic rat dorsal root ganglia neurons to Clostridium botulinum neurotoxins. Toxicon 38: 245-258, 2000.

11. Pieper C: Botulinum toxin type a neuromuscular blockade in the treatment of lower extremity spasticity in cerebral palsy: a randomized, double-blind, placebo-controlled trial. Pediatr Phys Ther 13: 92-94, 2001.

12. Jones OM, Moore JA, Brading AF and Mortensen NJ: Botulinum toxin injection inhibits myogenic tone and sympathetic nerve function in the porcine internal anal sphincter. Colorectal Dis 5: 552-557, 2003.

13. Brashear A, Lew MF, Dykstra DD, Comella CL, Factor SA, Rodnitzky RL, Trosch R, Singer C, Brin MF, Murray JJ, et al: Safety and efficacy of NeuroBloc (botulinum toxin type B) in type A-responsive cervical dystonia. Neurology 53: 1439-1446, 1999.

14. Adachi M, Otsuki M, Akatsu M and Tase C: The effects of heat stimulation and cold stress on the rats with cervical sympathectomy. Masui 52: 1293-1299, 2003 (In Japanese).

15. Huntoon MA: The vertebral artery is unlikely to be the sole source of vascular complications occurring during stellate ganglion block. Pain Pract 10: 25-30, 2010. 